(C) 1996 IEEE. Personal use of this material is permitted. However, permission to reprint/republish this material

for advertising or promotional purposes or for creating new collective works for resale or redistribution to servers

or lists, or to reuse any copyrighted component of this work in other works must be obtained from the IEEE.

\title{
The Safety Interlock System of Synchrotron Radiation Research Center
}

\author{
T. F. Lin, J. P. Wang \\ Synchrotron Radiation Research Center, Taiwan, R. O. C.
}

\begin{abstract}
The Safety Interlock System (SIS) at the Synchrotron Radiation Research Center (SRRC) is introduced. SIS is designed to protect people from exposure to accidental high radiation. It is composed of three subsystems. The Access Control System (ACS) prevents people from being exposed to the extremely high radiation inside the booster and storage ring shielding housing (or called the interlock area) during machine operation. The Beamline Enable Controller (BEC) controls the operation of the main shutter of each beamline. The Hutch Interlock System (HIS) prevents users from exposed to the radiation present when the X-ray bemlines are operating. The interlock design principles, the system features, the safety logic and electronic circuits are described along with the operation procedures.
\end{abstract}

\section{Introduction}

The Safety Interlock System (SIS) is designed to protect personnel from exposure to radiation present when the synchrotron facilities are operating. SIS is a system that ensure certain conditions have been met, in a proper sequence, before the accelerator can be operated, the beam can be injected to the storage ring or a beamline can be used. In addition, any action that could potentially cause accidentally exposure will violate the SIS system and cause a fault. The result of a SIS fault will terminate the booster operation, cease the injection process, and eliminate all the stored electron beam in the storage ring.

SIS consists three subsystems. The upper part of SIS is the Access Control System (ACS). This system controls the operation of booster and storage ring. Access control, automatic broadcasting, search confirmation, injection warning, and emergency shut down are the basic functions of ACS. The middle part of SIS is the Beamline Enable Controller (BEC) which controls the open mechanism of the main shutter of each beamline. The lower part of SIS is the Hutch Interlock System (HIS). This system safeguards the operation of the wiggler X-ray beamline. The interlock design principles are set following the guidance of the up-to-date accelerator safety order[1] [6]. The logic development, prototype construction, and real system installation are executed by SRRC staff. The system has been reviewed by the Safety Committee of SRRC and outside expertise.

\section{Interlock Design Principles}

The merit of an appropriate beam interlock system not only increases the degree of protection afforded individuals, but also reduces the technical and administrative burden. The fundamental philosophy is to make sure fail safe operation of the system, and to provide back-up functions in the event of failure. SIS at SRRC is designed following the principles given below.

1. The system should be fail safe, redundant, unrestrictive, and testable.

2. Emergency-off and search confirmation buttons should be provided inside the interlock area, and be clearly visible, easily distinguishable, unambiguously labeled, and readily accessible. 3 . Emergency exit mechanisms should be provided in entrance doors of interlock area, even when interlocked. Emergency entry features for interlocked doors are not precluded.

4. Clearly labeled status indicators reflecting actual conditions should be provided at entrance doors.

5. A reasonable search interval should be provided for each interlock area. An audible and visual warning interval should also be provided before the beam is introduced.

6 . The main shutter of each beamline should be closed during injection, and the branch shutters (photon shutters) should not be opened before the open of the main shutter.

7. Once SIS fault is happened, there should have redundant devices to cease the injection process and dump all the stored electron beam.

\section{System Description}

SIS can be classified into three different control levels. The upper part is the ACS of the booster and storage ring. BEC and HIS are the middle and lower part respectively. All these three systems are tied into the injector and ring control systems. Any violation of these systems will cease the injection process and dump the stored beam. The principal components and operation logic of each subsystem are described in the following section.

\section{Access Control System}

ACS controls the operation of the booster and storage ring. The block diagram of this system is shown in Fig. 1. The principal components together with their functions are describedbelow.

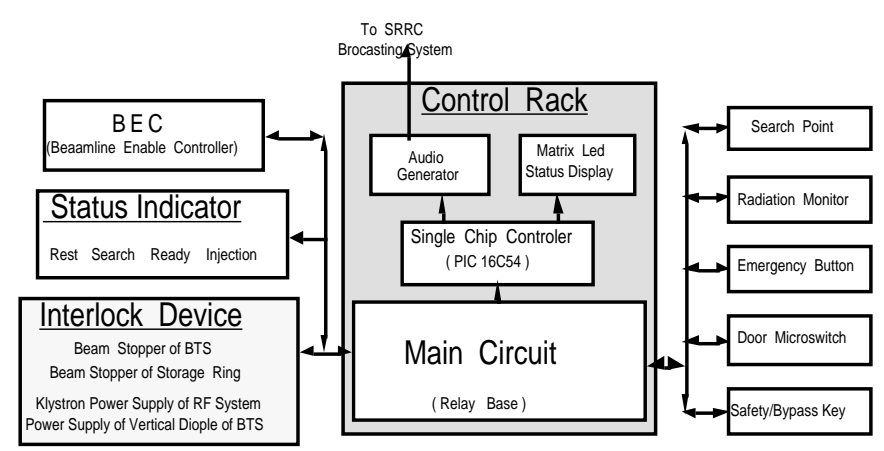

Fig 1 Accesss Control System (ACS) block diagram 


\section{(1). Control Rack}

This is the main body of ACS. Signals from door microswitches,search points, emergency buttons, radiation monitor, safety key and bypass key are processed here. Any emergency action been detected, it will send a command to the interlock devices to cease the operation of the machine. Status indication, automatic broadcasting, injection alarm and feedback monitor functions are also provided by this hardwired relay based control rack.

\section{(2). Status Indicator}

Status indicators are installed at entrance doors of interlock areas and some suitable positions to show the operation condition of the booster and storage ring. Each indicator receives command from the control rack and is able to show 4 different modes of operation.

Rest : Green light is shown in indicator. Beam is off, electrical hazard is also off.

Search : Yellow light is shown in indicator. Search procedure is going to be taken.

Ready : Red light is shown in indicator. Search is completed. Beam may be stored.

Injection : Flash red light is shown in indicator. Injection process is undertaking.

\section{(3). Search Point}

Search points are installed inside booster shielding room and storage ring shielding tunnel. Every search point should be activated sequentially in the search procedure. Violate the sequence or over the given search time period will cause the search invalid.

\section{(4). Emergency Button}

Emergency buttons are installed inside the interlock areas with clearly visible indicator and easily distinguishable color and labeling. Once someone being trapped inside the interlock area, emergency button provides the function to shutdown the machine operation immediately.

\section{(5). Force In/Out}

Force in/out mechanism is provided at every entrance door of the interlock areas. Should serious accident be happened, anyone could use the force in/out function to get in/out the interlock area to save the personnel or equipment in time. In ACS, force in/out process is treated as an emergency event.

\section{(6). Interlock Device}

The interlock devices linked to ACS are shown in table 1 . Anyone of these devices can cease the operation of the injector, terminate the injection process and eliminated the stored beam. This configuration can fulfill the redundant requirement.

\section{Table 1. Interlock Devices of ACS}

\begin{tabular}{cc}
\hline Booster ACS & Storae Ring ACS \\
\hline Gun Trigger of LINAC & Beam Stopper of BTS \\
\hline Gun High Voltage of LINAC & Beam Stopper of Storage Ring \\
\hline Modulator High Voltage of LINAC & Klystron Power Supply of RF System \\
\hline Klystron Power Supply of RF System & Power Supply of Vertical Dipole of BTS \\
\hline
\end{tabular}

\section{Beamline Enable Controller}

BEC is located at the control room of storage ring and is controlled by the operator. It governs the operation of the main shutter of each beamline. Any one of the beamlines should get the enable signal issued from this device before it can lift the safety shutter and introduce the synchrotron radiation to the end station. When HIS fault is sensed, it will send a signal to ACS to turn off the klystron power supply of the storage ring RF systems. The stored beam will be eliminated in this situation.

The operation of BEC is interlocked with the two beam stoppers of the transport line (BTS). It will keep in disable state when any one of the beam stoppers of BTS is open. This design insures that no main shutter of beamlines will be open during the injection process. The high intensity injection caused bremsstrahlung radiation will always be shielded by the heavy metal shutter. Radiation protection objective can be achieved.

\section{Hutch Interlock System}

The design philosophy of HIS is nearly the same as that of ACS. However, its scale is reduced just to cover the area of a hutch. Most of the components described in ACS section are used in HIS. The introduction given below will focus on the unique features of HIS.

The electronic circuit of HIS is shown in Figs. 2. The final target of HIS is to open the photon shutter of the beamline. Before reaching this goal, enable signals issued from other systems should be received, emergency chain should be closed, search procedure should be performed, hutch door should be locked, hutch key should be returned to control panel, audible and visual alarm interval should be finished, and user control button should be activated. The enable signals are linked directly to the user control button and are independent with hutch related circuit. This make it possible to lessen the boring and unnecessary search procedure in the re-injection event after unexpected beam dump.

A safety key and a test key are also designed in HIS. The safety key controls one of the enable signals of the photon shutter. It is administered by the safety person. Not before all the safety concerns of the beamline being solved, can the safety key be delivered to enable user control buttom. The test key is designed for function testing. Through a safety application procedure, users can get the key to test the function of the photon shutter.

Once HIS fault is happened, i.e. emergency button been activated or hutch door been forced open, a signal will send to BEC for closing the main shutter and dumping the stored beam. A manual reset process is required before the hutch can reopen for synchrotron radiation experiment.

\section{Operation Procedure}

Access to the interlock area is restricted, so as to prevent accidental operation, by means of mechanical locks, micro- 
switches, search points and control keys. A search procedure is also needed for confirming the safe conditions inside the interlock area. Except for some insignificant differences, the procedures for accessing and searching the interlock area are same for both ACS and HIS. Followings are the summaries of HIS operation procedures.

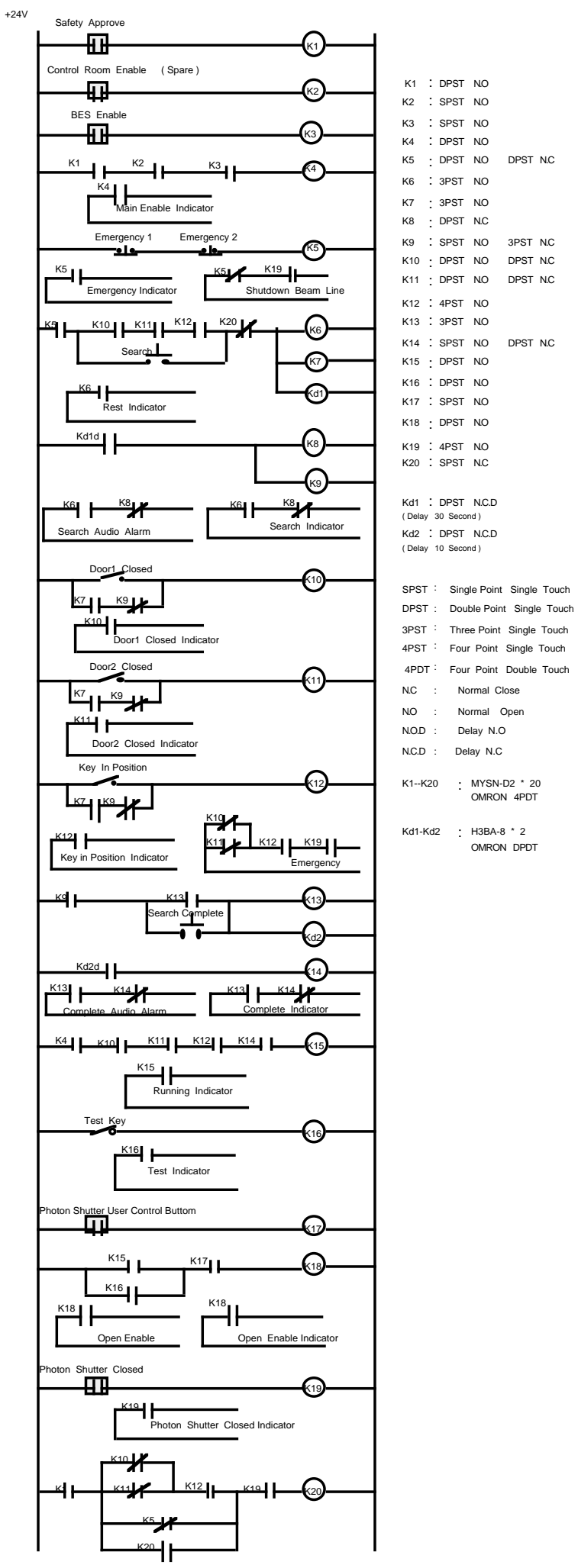

1. Close photon shutter using the user control button on the control panel. Turn hutch key to "release" position and pull it out.

2. Open the hutch door using hutch key. When hutch door is open, photon shutter is disable. Users can work safely in the hutch.

3. After works being done, push the search point inside the hutch. This action activates a flashing lamp and an audible buzzer. A timer is also activated to stop the warning process and give user a predetermined time $(30-60 \mathrm{sec})$ to search hutch, lock hutch door, return hutch key back to control panel, and turn it back to "lock" position.

4. Failure to comply with previously described action will cancel the procedure, search process must be re-tried.

5. Push the "search complete" button on the control panel. This action turns off the light inside the hutch for 10 to 15 seconds.

6. After the search process, the hutch is ready for beam on. If someone is still imprisoned in the hutch, he can force open the door or push the "emergency" button to cancel the open of photon shutter and dump the stored beam.

\section{Summary}

The safety interlock system at SRRC is established by the cooperation of instrument and control experts and safety people. The design logic of this system is fulfilled with the safety interlock principles. The successfully implementation of this system has increased the operation safety of SRRC facilities.

\section{References}

[1] Department of Energy, "Accelerator Safety Order, Guidance", (1991).

[2] T. Dickinson, "Radiation Safety Systems at the NSLS", Proceedings of the 1987 IEEE Particle Accelerator Conference, 1605-1607, (1987).

[3] Y. Satow, T. Kosuge and T. Matsushita, "Radiation Safety Interlock System for Photon Factory X-ray Beam Lines", Nucl. Instrum. Meth., Sect. A., (1986).

[4] E. Benson, K. Crook, N. Ipe, G. Nelson, J. C. Liu, J. Cerino, R. Hettel and R. Yotam, "The Personnel Protection System for a Synchrotron Radiation Accelerator Facility : Radiation Safety Perspective", SLAC-Pub-5833, (1992).

[5] R. C. Mccall, W. R. Casey, L. V. Coulson, J. B. Mccaslin, A. J. Miller, K. F. Crook and T. N. Simmons, "Health Physics Manual of Good Practices for Accelerator Facilities", SLAC-Report-327, (1988).

[6]. K. Batchelor, "National Synchrotron Light Source Safety Analysis Report", BNL 51584, UC-28, (1982).

Fig 2 Hutch Interlock System (HIS) electronic circuit 